\title{
ANÁLISIS DE LA FORMACIÓN DE LAS ENFERMERAS I. DE LA INSTITUCIONALIZACIÓN DE LAS ENSEÑANZAS SANITARIAS Y SU TEORÍA CURRICULAR
}

\author{
Ana M. PIERA Orts \\ Lic. Cc. de la Educación \\ Hospital General Universitario de Valencia \\ Correspondencia: Dr. Lluch, 159-1 ${ }^{\circ} .46011$ Valencia
}

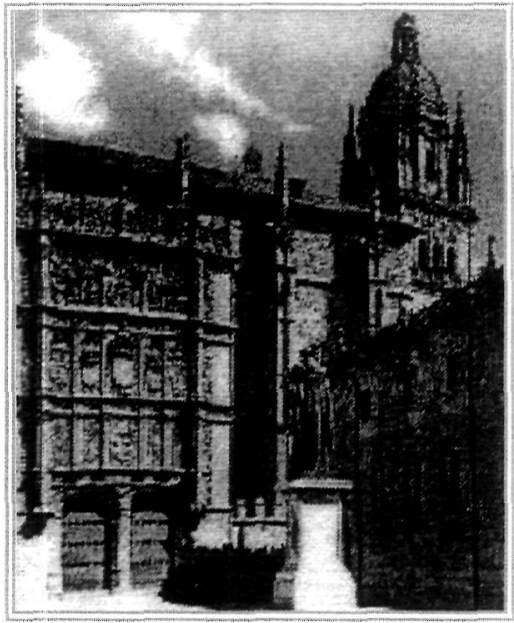

Universidad de Salamanca

\section{RESUMEN}

En este artículo se muestra el modo en que se estableció una vía de acceso a la indagación del problema seleccionado -el proceso de formación enfermera y la adecuación a de éste a las necesidades de su ejercicio profesional- a través de la institucionalización de las enseñanzas sanitarias, la revisión de los documentos que se han producido durante su más reciente construcción curricular, tras su inclusión en el ámbito universitario, así como de las imágenes que posee la enfermera sobre el currículum enfermero.

\section{THE EDUCATION OF REGISTERED NURSES: A DIAGNOSIS II. THE INSTITUTIONALIZATION OF HEALTH TEACHING AND THE CURRICULUM THEORY}

\section{SUMMARY}

This paper investigates the current conditions of the question undar study -the process of nursing education and how it meets the requirements of the profession- in order to obtain data on the institutionalization of health teaching, the document revised during the latest curriculum grafted following is being included as a university subject and the opinions of nurses on the nursing curriculum.

\section{INTRODUCCIÓN}

La cuestión de la formación básica de las enfermeras aparece ante la opinión pública como una cuestión irrelevante, dado que tradicionalmente se ha venido concediendo mayor atención a la relación médico-paciente que a la de enfermera-paciente, como efecto directo del ejercicio del médico que es el principal legitimador del papel de enfermo
(ABERCROMBIE, HILL y TURNER, 1992). Por esta misma razón, igualmente, parece que el diagnóstico y tratamiento médico de las dolencias han tenido una mayor supremacía que los cuidados terapéuticos enfermeros, a la vista de los efectos que los primeros han causado tanto sobre los modos organitativos sanitarios como sobre las expectativas de los propios pacientes. En esencia, dentro de los 
ámbitos sanitarios, no ha llegado a ser considerada tema de estudio oportuno, esta formación básica, a la vista de la prácticamente inexistencia de trabajos de evaluación de programas de formación enfermeros, como se detectó a partir de la búsqueda documental. Un tema, que, por su especificidad formativa y por el uso de técnicas clínicas que requiere este tipo de profesionales, a pesar de ser una profesión de servicios, tampoco ha tenido cabida especial en el ámbito de estudios de modelos formativos universitarios, como en el que ahora nos hemos introducido.

Desde la óptica del análisis institucional que parte de que la realidad se construye socialmente (BERGER y LUCKMANN, 1994), tratamos de acercarnos hacia el material con que se construyen las legitimaciones que mantienen este universo, descrito desde la revisión del proceso histórico de socialización de la actual diplomatura de enfermería, así como el desarrollo del análisis de la institucionalización de las enseñanzas enfermeras y su construcción curricular.

\section{DE LA INSTITUCIONALIZACIÓN} DE LAS ENSEÑANZAS SANITARIAS Y LA CONFIGURACIÓN HISTÓRICA

\section{DE LA FORMACIÓN ENFERMERA}

Para acercarnos a este proceso fuimos siguiendo el hilo conductor del modo en que se produjo la configuración histórica de esta profesión, cuál era la tradición académica enfermera en nuestro país, buscando la referencia original de la enfermera acreditada con estudios que enlaza, directamente, con nuestra actual enfermera universitaria, cuál había sido el marco político-administrativo de las regulaciones curriculares en las etapas históricas más recientes que habrían afectado a los actuales diseños formativos y el contenido, así como cómo códigos de su construcción curricular.

En una primera parte del recorrido histórico, se procuró que los fragmentos de historia recogida mantuviesen conexiones evolutivas para facilitar el desarrollo de la estructura teórica que se presenta. Esos saltos en la historia venían dados por el solapamiento que se produce entre algunas cuestiones que nos sirvieron como recurso de análisis, pues si queríamos detectar qué sistematización o qué regulaciones, a través de un período histórico, que fundamentalmente recogíamos a partir del siglo XIX, en ocasiones tendríamos que saltar de las organizaciones académicas a las políticas, e incluso de un período histórico a otro, en razón al seguimiento de un aspecto puntual, como pudiera ser la incorporación de una disciplina académica o la modificación del tipo de ocupaciones a que se dedicaban diferentes grupos de personas en las instituciones hospitalarias de encierro.

En una segunda parte, modificamos ese estilo de recopilación de datos históricos por una secuencia lineal que, a partir de documentos de diferente procedencia, nos permitió establecer el mapa de intereses en la negociación curricular enfermera desde 1970, evitando así la peligrosidad de abordar un tema a partir de «instantáneas» sobre acontecimientos singulares, y así este bloque nos permitió actuar como «semillero» para el análisis teórico que nos ocupaba.

De su análisis tratamos de obtener la naturaleza de las coaliciones que respondían tanto a la estructura de los intereses materiales y del discurso, como a los diferentes climas para la acción, es decir, cómo se manifiestan las perspectivas profesionales asociadas, el status, los grupos de materias académicas o la asignación de las disciplinas a los Departamentos correspondientes.

Respecto al concepto de enfermedad, no cabe duda que su concepción ha incidido sobre la configuración de las enseñanzas médicas y en el caso de enfermería, este efecto lo localizamos en las dos últimas décadas, en la tendencia a sustituir el modelo médico-biológico-somático por el modelo del proceso de atención enfermera. El primero, basado en las aplicaciones terapeúticas determinadas por el médico; y, el segundo, basado en valoraciones enfermeras sobre el paciente desde una perspectiva psico-somática y los cuidados que precisa, además de las aplicaciones terapeúticas. Aunque esta diferenciación no deja de ser una construcción social, marcada por un interés en la diferenciación profesional, no siendo propiamente la salud y enfermedad lo que adquiere más relevancia a este debate, si- 
no la dirección que tome el concepto de profesional de enfermería, como veremos más adelante, cuando a través de distintos documentos que revisamos, se nos mostraron los diferentes intereses de las organizaciones profesionales.

Desde la perspectiva histórica se pueden esbozar a partir de unos criterios de selección de contenidos, algunos mecanismos de institucionalización de las enseñanzas sanitarias, como modo de acercarnos a la estructura del currículum. Un criterio de seleción, lo podemos observar en el plan Mata durante el siglo XIX, por la utilidad que se considera que poseen en ese momento, las disciplinas de Higiene Pública o Toxicología, para el propio gobierno, lo que imprimió un estilo propio en los currícula de este período.

Un segundo criterio de selección es la forma de crecimiento del conocimiento en las ciencias de la salud, y de su posterior desarrollo tras la observación microscópica, lo que llevó parejo el establecimiento de «divisiones» (BARONA, 1990) en el estudio de las patologías, como especializaciones del saber médico y por tanto, conocimiento parcelado de la enfermedad, estrechamente vinculado a la organización de los servicios médicos, parámedicos y enfermeros de las organizaciones sanitarias, a las que llegan los profesionales con un grado de socialización adquirido en las instituciones sanitarias, donde se atenderán a los pacientes según patologías. Esta división en la patología, es la que permite que no se cuestione de forma explícita la organización hospitalaria de unidades de atención al paciente, en función de los cuidados que requieren, en lugar de su agrupación por especialidades médicas, en cuyo trasfondo conviven razones de jerarquía profesional y de carácter económico por la rotación médica que supondría.

Un tercer criterio de selección de contenidos gira en torno a la necesidad de incorporación de nuevas disciplinas que crecen paralelamente al avance técnico, como es el caso de la quimioterapia, farmacología experimental o las técnicas quirúrgicas. Y por último, el desarrollo y la aceptación de procesos psíquicos que intervienen en los casos patológicos, que hacen considerar en la actuación de tratamiento, la dimensión psicológica.
Estos cuatro criterios que influyen sobremanera en la construcción curricular, forman parte de un enrejado sobre el que se mueven factores sociales $y$ profesionales de forma enlazada. Téngase en cuenta que si bien, la socialización del profesional sanitario parte de la institución académica donde se sancionan los títulos que posibilitan el ejercicio profesional (BARONA, 1990, p. 212), es la institución académica, la que de forma habitual, suele ir recogiendo las novedades sobre el conocimiento generado a partir del ámbito de la práctica profesional para incorporarlas a sus currícula.

Una cuestión relevante, en cuanto a la incorporación de otros profesionales al ámbito de la salud en el territorio español, fue la propia evolución de las estructuras hospitalarias que, parejo al desarrollo de técnicas quirúrgicas que prosperaban a partir del nacimiento de la bacteriología y la inmunología, permitieron la potenciación de los hospitales, dado su aumento de eficacia y por tanto su nueva consideración social, lo que contribuyó a la incorporación de otros profesionales como es el caso de practicantes, enfermeras, comadronas, etc. a estas organizaciones, ya dentro del siglo $\mathrm{XX}$, aunque anteriormente existiesen los llamados hospitaleros o religiosas sin ninguna formación (BARONA, 1990, p. 234).

\section{DE LA APARICIÓN DE LA ENFERMERA EN LA ESTRUCTURA ACADÉMICA}

La Real Orden de 21 de noviembre de 1861 regulaba la formación de practicantes y matronas, cuyos estudios se podían cursar en las Universidades de Madrid, Barcelona, Granada, Santiago, Sevilla, Valencir y Valladolid (HERRERA RODRÍGUEZ, 1989).

El Decreto de Ruiz Zorrilla de 21 de octubre de 1868 , año en que iniciaba el período del sexenio revolucionario, autorizaba la libertad de enseñanza: lo que dio lugar a la creación de diversos centros de enseñanzas para formación sanitaria. Posibilidad que permitió la formación de profesionales sanitarios, en diferentes disciplinas que hasta ese momento no se contemplaban en los programas de formación de carácter oficial. Estas disciplinas tuvieron una orientación científica, como es el caso del centro creado en Sevilla por Federico Rubio, la 
«Escuela Libre de Medicina», en la que se potenció la medicina experimental. En Madrid se creaba la «Escuela Práctica Libre de Medicina y Cirugía» en la que se potenciaron los estudios de disciplinas básicas como fisiología, histología o histoquímica, con carácter experimental y comparado. A partir de este momento y diferenciadas de forma no institucional en el conjunto de disciplinas, se iniciaría la transformación en el conocimiento, hacia las especialidades y por tanto hacia las especializaciones profesionales y reconocimiento académico de especialidades como ginecología, urología, etc.

Fue un proceso en el que los diferentes titulados solicitaron las homologaciones a titulaciones autorizadas en los últimos planes de estudio y así en 1885 se celebraba un congreso en Barcelona, en el que los llamados ministrantes, solicitaron la homologación de su título con el de los médicos, con la ampliación de estudios pertinente. Los estudios de practicante, que ya habían sido objeto de regulación en 1861 , se habían vuelto a revisar en 1868 , pero no como una titulación diferente en función de la universidad, como vimos que sucedía con el Plan Mata o el de Bravo Murillo, sino como una profesión sanitaria, distinta a la del médico.

La enseñanza para matronas y practicantes se reguló nuevamente en 1888 por una disposición siendo en 1895 completada con una Real Orden cuyo contenido se ampliaría con un Decreto en 1901. Dentro del conjunto de profesiones sanitarias, la figura de la enfermera se hace patente a partir de la creación de la primera escuela para enfermeras con la denominación de «Santa Teresa de Hungría» en 1895, dentro del «Instituto de Terapéutica Operatoria» fundado por Federico Rubio, un efecto indirecto de que la gran mayoría de las reformas estuvieron en función de las decisiones de naturaleza política, como por ejemplo, las que guardaban relación directa con la promulgación de la Instrucción General de Sanidad en 1904, que regulaba y reconocía, entre otras cuestiones, como profesiones sanitarias, la de practicante (BERNABEU MESTRE y COTANDA SANCHO, 1991); por cierto, una de las múltiples manifestaciones de la separación profesional derivada del género.

\section{DE LOS PROGRAMAS ACTUALES}

Desde la promulgación de Ley General de Educación de 1970 hasta la elaboración de las últimas directrices generales para los planes de estudio se ha producido una larga cadena de documentos y acciones encaminadas a la reformulación de los planes de estudio de enfermería. De las instancias político-administrativas y las organizaciones colegiales, así como otros colectivos se han recogido gran cantidad de fuentes documentales que en este artículo no tienen espacio y, por tanto, sólo apuntaremos lo más aclaratorio.

Sirva como ejemplo del análisis documental efectuado, la reconstrucción de una situación puntual a través del contenido de una serie a de escritos oficiales. En Octubre de 1990, las aspiraciones que el Consejo General de Colegios de Diplomados en Enfermería (CGCDE) veía satisfechas sus demandas, según se recoge del texto del escrito firmado por el Secretario de Estado de Universidades e Investigación (CARTA, 2259, 23.10.90), quien en el punto segundo de este escrito afirma que las modificaciones propuestas por el Consejo General, "salvo mejor entendimiento, parecen centrarse solamente en dos puntos relevantes: la garantía sobre el reconocimiento del título por parte de la Comisión de las Comunidades Europeas y, en segundo lugar, la exclusividad del área de conocimiento de Enfermería en algunas materias troncales".

Un largo recorrido que nos muestra, en términos de teoría sustantiva, el ajuste a un orden negociado, donde las partes implicadas fueron conjugando todas sus estrategias al objeto de encontrar un punto de acuerdo sobre el que poner en marcha los diferentes currícula de Enfermería. En esa negociación, van apareciendo todas aquellas cuestiones de fondo que afectan a la profesión enfermera y a la organización de servicios sanitarios que tan directamente inciden en la formación de las enfermeras.

\section{DE LA TEORÍA CURRICULAR EN ENFERMERÍA Y SU CONFIGURACIÓN COMO UNA CONSTRUCCIÓN SOCIAL}

Para esta sección se revisaron las concepciones localizadas sobre el currículum en el área enferme- 
ra, para conocer en qué medida la reflexión sobre el currículum tiene un espacio en sus propios ámbitos docentes de formación profesional y cómo queda reflejada de un modo implícito en su actividad. Desde luego, esta revisión se llevó a cabo a partir de un análisis posicional para ubicar al lector en la perspectiva curricular del proceso y no del producto.

Desde esta situación, parece que para empezar un estudio de la teoría del currículum, es necesario también empezar un estudio de metateoría del currículum, dado que aportar o no, una definición del currículum es para Kemmis y Fitzclarence, una toma de posición sobre lo que significa definir y lo que significa teorizar (KEMMIS y FITZCLARENCE, 1986).

Tal vez la dificultad teórica, que se presenta para Kemmis y Fitzclarence, queda aclarada por la forma de pensar el currículum que puede orientar la búsqueda de la expresión de su práctica, atendiendo a que:

«El currículum es una práxis antes que un objeto estático emanado de un modelo coherente de pensar la educación (...).Es una práctica en la que se establece un diálogo, por decirlo así, entre agentes sociales, elementos técnicos, alumnos que reaccionan ante él, profesores que lo modelan, etc. Desarrollar esta acepción del currículum como ámbito práctico tiene el atractivo de poder ordenar en torno a este discurso las funciones que cumple y el modo como las realiza, estudiándolo procesualmente: se expresa en una práctica y toma significado dentro de una práctica en alguna medida previa y que no sólo es función del currículum, sino de otros determinantes. Es contexto de práctica al tiempo que contextualizado por ella.» (GIMENO, 1988, p. 16).

Presentar brevemente el recorrido que se realizó a través de este ámbito, supone dejar de lado gran número de autores que aquí no podemos presentar. Pero hacemos un recorrido en bloque para mostrar, al menos, lo que define el ámbito del análisis curricular en enfermería. Por ejemplo, podemos citar que una de las reflexiones más reiteradas es la que reclama que la forma de introducir la investigación en enfermería, es a través de la formación del alumnado en esta línea (SWANSON, BOSSERT, BURGUESS, LONDON, REISNIG y ZIMMERMAN, 1988).
Junto a las afirmaciones que hemos recogido, aparecen otro tipo de trabajos del ámbito curricular en base al ejercicio docente enfermero, tales como el referido a las experiencias de formación enfermera en China (DESANTIS, 1988); Australia (ROBERTS, 1978) o en Israel (ZIV y DAPHA, 1989) cuyos contenidos exponen, respectivamente, la preparación de enfermeras instructoras para la organización de programas de educación, con estructura similar al Bachillerato en Artes de EE.UU.; la secuencia de programación educativa al estilo de Gagne o, finalmente, la re-estructuración de los programas de formación enfermera en su trasvase a la educación de nivel universitario.

Otros trabajos hacen referencia a los modelos de educación en enfermería, señalando que sus contenidos no están nivelados con los existentes en las escuelas semiprofesionales o en la universidad (FITZPATRICK, 1985); o se trata de reflexiones en torno al bajo nivel de conocimiento científico de un alto porcentaje de enfermeras registradas -un $60 \%$ (ROGERS, 1985); la posibilidad de promover unas prácticas de laboratorio que permitan la adquisición de un aprendizaje con mejores recursos (INFANTE, 1985); o incluso a los aspectos legales que garanticen la no discriminación, etc... a las nuevas necesidades de la enfermería del futura (DUMAS, 1985).

En 1986 la N.N.L. presentaba un conjunto de trabajos relacionados con la situación de enfermería de aquellas fechas (GOLDBERG, 1987; SHERMAN, 1987, DONLEY, 1987) y sus perspectivas de futuro (DUFFY, 1987; MITCHELL, 1987, INFANTE, 1987, FARLEY, 1987a; FARLEY 1986b).

Como muestra de los temas que preocupan a un buen número de profesoras de enfermería norteamericanas recogemos los que presentan la necesidad de modificar el currículum (DONLEY, 1989; MOCCIA, 1988; WATSON, 1988; AROSKAR, 1988), o la preocupación por promover una serie de experiencias que relacionen más directamente la teoría enfermera con la práctica asistencial (CLAYTON y MURRAY, 1989; DIEKELMANN, 1989; MARTIN, 1989; INFANTE, 1985). Aparece una constante preocupación sobre los temas relaciona- 
dos con la construcción teórica de los modelos de formación enfermeros, es decir, por todo aquello que permita exponer cómo se forma a la enfermera y para qué función, recogiendo tanto teorías educativas como modelos de gestión hospitalaria y tratando de configurar una forma de presentar la labor docente (FITZPATRICK, 1985; BOYD, 1988; O'LEARY, 1985; FARRELL, 1987; CHINN, 1989; ROGERS, 1985; SULLIVAN, 1986).

Otro planteamiento es el futuro de la profesión de enfermería, dado el coste de las prestaciones sanitarias que situarán a la enfermera en diferentes instituciones de niveles diferenciados, lo que promueve la reflexión sobre el futuro institucional para el que hay que prepararse (DONLEY, 1986; AMOS, 1985; BROWN, 1987; GREENLEAF, 1988; LANE, 1985; MUNHALL, 1988; DUMAS, 1985), así como la especialización (DUMAS, 1986; SHERWEN, 1985; JACKSON, B.S., 1986; POLLOK, 1986; GARNER, 1986; CARY, 1986; JOEL, 1986), lo que comporta una revisión de la acreditación de las titulaciones (PIEMONTE, 1985; LENN, 1986; CIPRIANO, 1985; DIAZ, 1985).

Sobre la configuración de los conocimientos básicos son escasos los trabajos y alguno de ellos es tan singular como el que propone establecer la necesidad de desarrollar una teoría dialéctica en la que entren los valores en juego y se promueba la discusión entre lo cualitativo y lo cuantitativo o aspectos como la condición humana, una especie de pupurrí que, en realidad, es un intento de apuntar hacia la necesidad de reflexionar sobre la práctica diaria (MOCCIA, 1985). Otros trabajos que revisan la configuración de los conocimientos son, generalmente, sobre el impacto de las nuevas tecnologías computerizadas en los hospitales y su efecto en las necesidades formativas (GROBE, 1985; HALES, 1985).

La cuestión de las implicaciones de todo este tipo de necesidades de cambio sobre el profesorado también es revisada (LINDEMAN, 1988; CORCORAN y TANNER, 1988; GOLDBLATT, 1986; WOOLLEY y COSTELLO, 1988) siendo todos los trabajos, orientados a plantear la necesidad de que los propios profesores reflexionen sobre su propia actividad educativa.
En general la preocupación por el «como»se trasmite y el «que» se desea forme parte del corpus teórico de los profesionales de enfermería es una constante que aparece en las publicaciones que hemos recogido. En el caso español, estas reflexiones se han producido hasta ahora, con escasa repercusión en las publicaciones especializadas, dada la situación de cambio que todavía vive esta profesión dentro de la estructura universitaria.

Los escasos trabajos sobre la teoría del currículum en enfermería, no llegan a aportar ninguna definición, que según Kemmis y Fitzclarence sería una toma de posición que no hemos constatado (KEMMIS y FITZCLARENCE, 1986), lo que nos habría permitido esclarecer qué tipo de selección de contenido y objetivos, qué organización de conocimientos y destrezas o qué métodos concernientes a cómo son seleccionados los contenidos, para ser secuenciados y controlados.

En suma, las publicaciones enfermeras nos muestran que el currículum es visto como un instrumento técnico para programar actividades educativas, que no penetra en la práctica real a través de los procesos reflexivos de los profesores, y queda instalado en el plano teórico de asesores y especialistas de la administración educativa o asociaciones profesionales, pero que, poco a poco, se percibe que irá instalándose en la práctica del profesorado, como se ve a partir de iniciativas de indagación (ALEXANDER, 1982 ).

Lo que se hace patente, a la vista de los modos organizativos de los conocimientos teóricos que actualmente se mantienen en los formatos curriculares enfermeros, es que mantienen, desde el inicio de su configuración como personal auxiliar médico y su posterior evolución como carrera profesional, una modalidad muy próxima al ámbito de éstos; en cuanto a la agrupación de contenidos en torno a las especialidades médicas. Difícilmente, se puede reelaborar una nueva imagen de la función y cometido profesional de la enfermera, si los planes de formación no se modifican sustantivamente en torno a un nuevo papel. Si el ejercicio profesional comporta una responsabilización en la puesta en marcha de elementos científico-técnicos adquiridos durante el 
proceso de formación, en buena lógica, cuando la estructuración de contenidos es tan similar a la de la figura del médico, pero en inferior cantidad, es de suyo natural, que de modo objetivo, se mantenga su subordinación en cuanto a la valoración técnica de cualquier ejecución profesional.

Llegados a este punto, hay que considerar el papel de las organizaciones profesionales, que en principio van a estimar que les corresponde, y sólo a ellas, esa valoración técnica; pero, cuando buscábamos qué orden negociado quedaba establecido en torno al currículum de enfermería en la etapa que más nos interesa ahora, es decir, desde que tiene estatuto de formación universitaria, comprobamos de nuevo una cierta dificultad, en cuya base se halla la escasa definición del papel que se asigna a la enfermera. Baste decir que la preocupación fundamental que constatamos durante largos procesos de discusión era la de añadir o suprimir disciplinas, casi siempre, de idéntico enunciado al que mantienen las de los médicos en su carrera profesional y la adscripción de dependencia departamental universitaria. Pareció quedar concluido parte de este proceso, cuando esa adscripción quedó bajo el Departamento de Enfermería; pero reparemos en que los enunciados de los contenidos teóricos siguen siendo de naturaleza médica, aunque precedidos de su condición enfermera como: enfermería médicoquirúrgica, enfermería psiquiátrica, enfermería geriátrica, farmacología, etc..., pero no conviene pasar por alto que muchas de estas ciencias consideradas como ciencias auxiliares tengan un carácter que, una y otra vez, se señalan dentro de una tendencia parcial dirigida hacia el conocimiento funcional más que descriptivo, como es el caso de la Anatomía. Esto explicaría ese sentido de las enfermeras como colaboradoras del médico.

\section{BIBLIOGRAFÍA}

ABERCROMBIE, N.; HILL, S.; TURNER, B. (1982) Diccionario de Sociología. Ed. Cátedra. págs. 285.

AMOS, L.K. (1985): Can Single-Purpose Institution Provide Baccalaureate. En: Patterns in Education: The Unfolding of Nursing. New York. Ed. National League for Nursing. págs. 41-48.
ALEXANDER, M.F. (1982): Nurse Education: An Experiment in Integration of Theory and Practice in Nursing. En: REDFERN, S.J.; SISSON, A.R.; WALKER, J.F. and WALH, P.A.: Issues in Nursing Research. London. The Macmillan Press Ltd. pags. 74-93.

AROSKAR, M.A. (1988): Curriculum Revolution: A Bioethical Mandate for Change. En: Curriculum Revolution: Mandate for Change. New York. De. National League for Nursing. págs.123-136.

BARONA VILAR, J.L. (1990) Introducció a la Medicina. Universitat de València. Servei de Publicacions. págs. 242.

BERGER, P.; LUCKMANN, T. (1994) La construcción social de la realidad. Amorrortu Editores. $1^{\text {a }}$ edición en castellano 1968. Buenos Aires. 233 págs.

BERNABEU MESTRE, J.; COTANDA SANCHO, S. (1991) Antecedentes históricos del proceso de socialización de la enfermería española. Desarrollo normativo (1904/1935). En: Enfermería Científica, núm. 111. Junio. págs. 28-36.

BOYD, C.O. (1988): Phenomenology: A Foundation for Nursing Curriculum. En: Curriculum Revolution: Mandate for Change. New York. De. National League for Nursing. págs.65-88.

BROWN, B.J. (1987): Strategic Educational Planning: A Giant Step into Our Future. En: Patterns in Nursing: Strategic Planning for Nursing Education. New York. National League for Nursing. págs. 153-160.

CARY, A. (1986): Specialization without Walls: Community-Based Nursing. En: Patterns in Specialization: Challenge to the Curriculum. New York. National League for Nursing. págs. 257-270.

CHINN, P.L. (1989): Feminist Pedagogy in Nursing Education. En:Curriculum Revolution: Reconceptualizing Nursing Education. New York. Ed. National League for Nursing. págs. 9-24.

CIPRIANO, P. F. (1895): Certification: Self-Regulation for Specialy Practice. En: Patterns in Specialization: Challenge to the Curriculum. New York. National League for Nursing. págs. 77-84.

CLAYTON, G.M., MURRAY, J.P. (1989): Faculty-Student Relationships: Catalytic Connection. En: Curriculum Revolution: Reconceptualizing Nursing Education. New York. Ed. National League for Nursing. págs. 43-54.

CORCORAN, S.A. y TANNER, C. (1988): Implications of Clinical Judgment Research for Teaching. En: $\mathrm{Cu}$ rriculum Revolution: Mandate for Change. New York. De. National League for Nursing. págs.159176. 
DESANTIS, L. (1988): Development of a nurse-instructor preparation program in China. En: Int. J. Nurs. Stud., Vol. 25, No. 2. págs. 135-146.

DIAZ, S.L. (1985): Assessing Single-Purpose Institutions through the Accreditacion Process. En: Patterns in Education: The Unfolding of Nursing. New York. Ed. National League for Nursing. págs. 65-70.

DIEKELMANN, N. L. (1989): The Nursing Curriculum: Lived Experiences of Students. En: Curriculum Revolution: Reconceptualizing Nursing Education. New York. Ed. National League for Nursing. págs.2542.

DONLEY, R. (1986) The Specialist in the Markeplace. Analysis of Supply and Demand. En: Patterns in Specialization: Challenge to the Curriculum. New York. National League for Nursing. págs. 1-6.

- (1987) Strategic Planning: A Rational Activity. En: Patterns in Nursing: Strategic Planning for Nursing Education. New York. Ed. National League for Nursing. págs. 13-22.

DONLEY, S.R. (1989) Curriculum Revolution: Heeding the Voices of Change. En: Curriculum Revolution: Reconceptualizing Nursing Education. New York. Ed. National League for Nursing. págs. 1-8.

DUFFY, M.E. (1987) Innovation as a Survival Strategic., En: Patterns in Nursing: Strategic Planning for Nursing Education. New York. National League for Nursing. págs. 47-70.

DUMAS, R.D. (1985) Mission for the Future of Nursing Education. En: Patterns in Education: The Unfolding of Nursing. New York. Ed. National League for Nursing. págs. $27-40$.

DUMAS, R.G. (1986) Challenging the Curriculum: The Future of Specialization. En: Patterns in Specialization: Challenge to the Curriculum. New York. National League for Nursing. págs. 27-40.

FARLEY, V.M. (1987a) Strategic Planning: An Organizing Framework for Nursing Education. En: Patterns in Nursing: Strategic Planning for Nursing Education. New York. Ed. National League for Nursing. págs. 3-12.

- (1987b) Creating Change: A Survival Strategy. En: Patterns in Nursing: Strategic Planning for Nursing Education. New York. National League for Nursing. págs. 143-152.

FARRELL, J. (1987) Recruitment Planning in Light of Changing Student Profiles. En: Patterns un Nursing: Strategic Planning for Nursing Education. New York. National League for Nursing. págs. 133-142.

FITZPATRICK, M.L. (1985) Perspectives on the Patterns of Nursing Education. En: Patterns in Education: The
Unfolding of Nursing. New York. Ed. National League for Nursing. págs.3-10.

GARNER, J.S. (1986) Infection Control Nursing: Challenge to the Curriculum. En: Patterns in Specialization: Challenge to the Curriculum. New York. National League for Nursing.,págs. 157-170.

GIMENO SACRISTÁN, J. (1988) El curriculum: una reflexión sobre la práctica. Madrid. Ed. Morata, S.A. págs. 414.

GOLDBERG, E. (1987) Nursing Education's Locus of Control: Where is the Future?. En: Patterns in Nursing: Strategic Planning for Nursing Education. New York. National League for Nursing. 23-34.

GOLDBLATT, K. (1986) Teaching the Teacher: Preparing the Educator for Specialists. En: Patterns in Specialization: Challenge to the Curriculum. New York. National League for Nursing. págs. 199-206.

GREENLEAF, N.P. (1988) Historical and Economic Perspectives on the Nursing Labor Force. En: Curriculum Revolution: Mandate for Change. New York. De. National League for Nursing. págs.107-122.

GROBE, S.J. (1985) The Impact of Computers on Nursing. En: Patterns in Education: Unfolding of Nursing. New York. Éd. National League for Nursing. págs. 91-100.

HALES, G.D. (1985) State-of-the-Art Technology: Computers and Curriculum. En: Patterns in Education: The Unfolding of Nursing. New York. Nationale League for Nursing. págs. 101-118.

HERRERA RODRÍGUEZ, F. (1989) La titulación de las matronas en la Facultad de Medicina de Cádiz durante el sexenio revolucionario (1868-1874). En: Toco-Ginecología Prática. vol. 48 núm. 539. págs. 230-36.

INFANTE. M.S. (1985) The Clinical Learning Experience: The Evolution of Nursing Work Force. En: Patterns in Education: The Unfolding of Nursing. New York. Ed. National League for Nursing. págs. 15-26.

- (1987) Nursing Educational Research: Planning to Achieve Our Future. En: Patterns in Nursing: Strategic Planning for Nursing Education. New York. National League for Nursing. págs. 107-116.

JACKSON, B.S. (1986) Specialization and Fragmentation: The Enterostomal Terapist. En: Patterns in Specialization: Challenge to the Curriculum. New York. National League for Nursing. págs. 271-278.

JOEL, L.A. (1986) Master's Prepared Caregivers in Line Positions: A Case Study. En: Patters in Specialization: Challenge to the Curriculum. New York. National League for Nursing. págs. 235-244. 
KEMMIS, S., FITZCLARENCE, L. (1986) Curriculum Therising: Beyond Reproduction Theory. Deakin University. 149 págs.

LANE, J.A. (1985) A Disease Called Egalitarism: SinlePurpose Institutions for Nursing. En: Patterns in Education: The Unfolding of Nursing. New York. Ed. National League for Nursing. págs. 57-64.

LENN, M.P. (1986) Accreditation: The State od the Art. En: Patterns in Specialization: Challenge to the $\mathrm{Cu}$ rriculum. New York. National League for Nursing. págs. 41-50.

LINDEMAN, C. (1988) Teaching Research in the Undergraduate Curriculum. En: Curriculum Revolution: Mandate for Change. New York. De. National League for Nursing. págs.231-247.

MARTIN, C. E. (1989) Alternatives for Students with life Experiences: Reconceptualizing Nursing Education. En: Curriculum Revolution: Reconceptualizing Nursing Education. New York. Ed. National League for Nursing. págs. 101-114.

MITCHELL, C.A. (1987) Future View: Nontraditional Education as the Norm. En: Patterns in Nursing: Strategic Planning for Nursing Education. New York. National League for Nursing. págs. 71-90.

MOCCIA, P. (1985) Dialectics of Theory Development. En: Patterns in Education: The Unfolding of Nursing. New York. Ed. National League for Nursing. págs. 71-78.

- (1988) Curriculum Revolution: An Agenda for Change. En: Curriculum Revolution: Mandate for Change. New York. De. National League for Nursing. págs.53-64.

MUNHALL, P.L. (1988) Curriculum Revolution: A Social Mandate for Change. En: Curriculum Revolution: Mandate for Change. New York. De. National League for Nursing. págs.217-230.

O'LEARY, J. (1985) Strategies for Recuiting Graduate Faculty in Nursing Service Administration. En: $P a$ terns in Education: The Unfolding of Nursing. New York. National League for Nursing. págs. 119-126.

PESET, M. y PESET, J.L. (1974) La universidad española. (Siglos XVIII y XIX). Despotismo ilustrado y revolución liberal. Madrid. Ediciones Taurus. págs.

PIEMONTE, R.V. (1985) Credentialing: The Thread Between Licensure, Acreditation and Certification. En: Patterns in Education: The Unfolding of Nursing. New York. National League for Nursing. págs. 127-142.
POLLOK, C.S. (1986) Regulating Advanced Nursing Practice: State Regulatory Boards. En: Patterns in Specialization: Challenge to the Curriculum. New York. National League for Nursing. págs. 51-66.

ROBERTS, K.L. (1978) Curriculum planning and the sequence of learning. En: The Australian Nurses'Journal. vol.7, núm. 8. págs. 40-42.

ROGERS, M.E. (1985) Nursing Education: Preparing for the Future. En: Patterns in Education: The Unfolding of Nursing. New York. Ed. National League of Nursing. págs. 11 14.

SHERMAN, S.E. (1987) Analyzing Past PerfomanceCreating a Better Future. En: Pattterns in Nursing: Strategic Planning for Nursing Education. New York. National League for Nursing. págs. 35-46.

SHERWEN, L.N. (1985) Specialty Core Curriculum: Competencies in Maternal-Infant Nursing. En: Patterns in Specialization: Challenge to the Curriculum. New York. National League for Nursing. págs. 147156.

SULLIVAN, T.J. (1986) Promoting Partnership: Education, Service and Specialization. En: Patterns in Specialization: Challenge to the Curriculum. New York. National League for Nursing. págs. 7-14.

SWANSON, M.J.; ZIMMERMAN, G.J.; BOSSERT, E.A.; BURGUESS, A.; CONDON, V; and REISWIG, J. (1988) Incorporating Research into Baccalaureate Nursing Education. En: Today's OR Nurse. vol. 10, núm. 10. págs. 31-36.

ZIV, L. and DAPHNA, L. (1989) Planning an Academic Nursing School in teh North of Israel. En: Nursing RSA Verplegind. vol. 4, núm. 1. págs. 32-34.

WATSON, J. (1988) A case Study: Curriculum in Transitión. En: Curriculum Revolution: Mandate for Change. New York. De. National League for Nursing. págs.1-8.

WOOLLEY, A.S. y COSTELLO, S.E. (1988) Innovations in Clinical Teaching. En: Curriculum Revolution: Mandate for Change. New York. De. National League for Nursing. págs.89-106.

\section{DOCUMENTOS E INFORMES}

SECRETARÍA DE ESTADO DE UNIVERSIDADES E INVESTIGACIÓN (1990): Carta núm. 2259. Dirigida al Presidente del Consejo General Colegios Diplomados Enfermería. 23.10. 90.

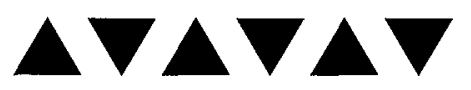

